TRANSACTIONS OF THE

AMERICAN MATHEMATICAL SOCIETY

Volume 315, Number 2, October 1989

\title{
INFIMA OF CONVEX FUNCTIONS
}

\author{
GERALD BEER
}

\begin{abstract}
Let $\Gamma(X)$ be the lower semicontinuous, proper, convex functions on a real normed linear space $X$. We produce a simple description of what is, essentially, the weakest topology on $\Gamma(X)$ such that the value functional $f \rightarrow \inf f$ is continuous on $\Gamma(X)$. When $X$ is reflexive, convergence of a sequence in this topology is equivalent to Mosco plus pointwise convergence of the corresponding sequence of conjugate convex functions.
\end{abstract}

\section{INTRODUCTION}

Over the last twenty years Mosco convergence of sequences of proper lower semicontinuous convex functions was held a prominent place in convex analysis in reflexive Banach spaces (see, e.g., [1]). To describe Mosco convergence, let $X$ be a real Banach space, and let $f, f_{1}, f_{2}, \ldots$ be proper, lower semicontinuous, convex functions on $X ;\left\langle f_{n}\right\rangle$ is declared Mosco convergent to $f$ provided both of the following conditions hold:

(i) for each $x \in X$, there exists $\left\langle x_{n}\right\rangle$ norm convergent to $x$ for which $f(x)=\lim _{n \rightarrow \infty} f_{n}\left(x_{n}\right)$;

(ii) whenever $\left\langle x_{n}\right\rangle$ is weakly convergent to $x \in X$, then

$$
f(x) \leq \liminf _{n \rightarrow \infty} f_{n}\left(x_{n}\right) .
$$

There are at least two different approaches to constructing topologies compatible with this notion of convergence $[1,5]$.

The high status accorded to Mosco convergence reflects its stability with respect to duality, as proved in finite dimensions by Wijsman [17], and in the reflexive setting by Mosco [13] (see also $[5,8,14,16]$ ). Specifically, $\left\langle f_{n}\right\rangle$ is Mosco convergent to $f$ if and only if the corresponding sequence of conjugate functions $\left\langle f_{n}^{*}\right\rangle$ is Mosco convergent to $f^{*}$. With respect to optimization, Mosco convergence leaves something to be desired. Although we can say that whenever $\left\langle f_{n}\right\rangle$ is Mosco convergent to $f$, that $\inf f \geq \lim \sup _{n \rightarrow \infty} \inf f_{n}$, we cannot say that $\inf f=\lim _{n \rightarrow \infty} \inf f_{n}$. For example, on the line, $\left\langle f_{n}\right\rangle$ is Mosco

Received by the editors April 25, 1988.

1980 Mathematics Subject Classification (1985 Revision). Primary 26B25; Secondary 49A27, 54B20.

Key words and phrases. Convex function, value functional, minimization, Mosco convergence, conjugate convex function, affine topology. 
convergent to $f$, where

$$
f_{n}(x)=\left\{\begin{array}{ll}
\infty & \text { if } x<0, \\
-x / n & \text { if } 0 \leq x \leq n, \\
-1 & \text { if } x>n,
\end{array} \quad f(x)= \begin{cases}\infty & \text { if } x<0 \\
0 & \text { if } x \geq 0\end{cases}\right.
$$

There is also an obvious lack of stability of the set of minimizers of $f$ with respect to the perturbed functions $\left\langle f_{n}\right\rangle$. Both flaws can be made to disappear with sufficient compactness assumptions. For example, if for all $n$ sufficiently large, $f_{n}$ has a minimum value in some common weakly compact set, it is not hard to show that inf $f=\lim _{n \rightarrow \infty} \inf f_{n}$, and that some subsequence of minimizers of the perturbed functions converges to a minimizer of $f$.

It is the purpose of this article to display what is, essentially, the weakest topology $\tau$ on the proper, lower semicontinuous, convex functions defined on a normed linear space $X$ (not necessarily reflexive or even complete) with respect to which the value functional $f \rightarrow \inf f$ is continuous (see Theorem 3.3 below). When $X$ is reflexive, we show that convergence of a sequence with respect to this topology amounts to Mosco plus pointwise convergence of the corresponding conjugate sequence.

\section{Preliminaries}

In the sequel $X$ will be a (real) normed linear space with continuous dual $X^{\prime}$. The collection of closed nonempty convex subsets of $X$ will be denoted by $\mathscr{C}(X)$. For an extended real valued function $f: X \rightarrow[-\infty, \infty]$, we define its epigraph and hypograph to be the following subsets of $X \times R$ :

$$
\begin{aligned}
\text { epi } f & =\{(x, \alpha): x \in X, \alpha \in R, \text { and } \alpha \geq f(x)\}, \\
\text { hypo } f & =\{(x, \alpha): x \in X, \alpha \in R, \text { and } \alpha \leq f(x)\} .
\end{aligned}
$$

We call $f$ convex (resp. closed) provided epi $f$ is a convex (resp. closed) subset of $X \times R$. It is well known that $f$ is closed if and only if $f$ is lower semicontinuous in the usual sense [6]. We call $f$ proper provided $f(x)$ is finite for some $x$, and for all $x, f(x)>-\infty$. In terms of epigraphs, this means epi $f \neq \varnothing$, and epi $f$ contains no vertical lines. We denote the proper, lower semicontinuous, convex functions on $X$ by $\Gamma(X)$. In particular, the indicator function $I(\cdot, C)$ of each nonempty closed convex subset $C$ of $X$, defined by

$$
I(x, C)= \begin{cases}0 & \text { if } x \in C \\ \infty & \text { if } x \notin C\end{cases}
$$

is in $\Gamma(X)$.

We next turn to the Young-Fenchel transform or conjugate operator; proofs of all the statements that follow in this paragraph can be found in [7, §14]. If $f \in \Gamma(X)$, we define the conjugate $f^{*}$ of $f$ (an extended real valued function on $X^{\prime}$ ) by the formula

$$
f^{*}(y)=\sup \{\langle y, x\rangle-f(x): x \in X\} \quad\left(y \in X^{\prime}\right) .
$$


The conjugate of $f$ is understood best as follows: $(y, \alpha) \in \operatorname{epi} f^{*}$ if and only if $f$ majorizes $x \rightarrow\langle y, x\rangle-\alpha$, so that the epigraph of $f^{*}$ parametrizes the continuous affine functionals majorized by $f$. The conjugate operator maps $\Gamma(X)$ onto the proper, convex, weak ${ }^{*}$-lower semicontinuous functions on $X^{\prime}$, which we denote by $\Gamma^{*}\left(X^{\prime}\right)$ in the sequel. We may define the second conjugate of $f$ by the formula

$$
f^{* *}(x)=\sup \left\{\langle y, x\rangle-f^{*}(y): y \in X^{\prime}\right\} \quad(x \in X) .
$$

With this convention, $f^{* *}=f$. Notice that there is no distinction between the first and second conjugate, provided $X$ is reflexive.

Our topology on $\Gamma(X)$ natural with respect to minimization is best approached from the epigraphical perspective. We think of convex functions on $X$ as subsets of $X \times R$, and topologize them using a topology on sets of subsets-a so-called hyperspace topology. We require some additional notation. Let $A \subset X$. We define the following subsets of $\mathscr{C}(X)$ associated with $A$ :

$$
\begin{gathered}
A^{-}=\{C \in \mathscr{C}(X): C \cap A \neq \varnothing\}, \quad A^{+}=\{C \in \mathscr{C}(X): C \subset A\}, \\
A^{\#}=\left\{C \in \mathscr{C}(X): \inf _{a \in A, c \in C}\|a-c\|>0\right\} .
\end{gathered}
$$

Notice within $C(X)$, we have $A^{\#} \subset\left(A^{c}\right)^{+}$, and $A^{\#}=\left(A^{c}\right)^{+}$provided $A$ is weakly compact. Historically, two of the three most prominent hyperspace topologies admit simple presentations in this notation: the Vietoris topology or finite topology is generated by all sets of the form $V^{-}$and $V^{+}$where $V$ is norm open, and the Fell topology or topology of closed convergence is generated by all sets of the form $V^{-}$where $V$ is norm open, and $\left(K^{c}\right)^{+}$where $K$ is norm compact. Deleting the word "norm", these definitions make sense for the closed subsets of an arbitrary topological space, rather than for the closed convex subsets of a normed linear space, and these hyperspaces have been thoroughly investigated at this level of abstraction $[9,10]$.

In [3], this author introduced a variant of the Fell topology on $\mathscr{C}(X)$, called the Mosco topology $\tau_{M}$, generated by all sets of the form $V^{-}$where $V$ is norm open, and $\left(K^{c}\right)^{+}$where $K$ is weakly compact. Clearly, the topology agrees with the Fell topology when $X$ is finite dimensional. Identifying functions with their epigraphs, we may view $\Gamma(X)$ as a topological subspace of $\left\langle\mathscr{C}(X \times R), \tau_{M}\right\rangle$, and by virtue of Lemma 1.10 of [12] and Theorem 3.1 of [3], Mosco convergence of sequences in $\Gamma(X)$ is compatible with $\tau_{M}$. Most importantly, Theorem 3.1 of [5] shows that Mosco's sequential continuity result for the Young-Fenchel transform in the reflexive setting may be strengthened to a bona fide continuity theorem (note: for reflexive $X, \tau_{M}$ is first countable if and only if $X$ is separable [3, Lemma 4.1]).

The function space topology of interest for our purposes here is closely connected to a second hyperspace topology, introduced by this author [4] in a (successful) effort to produce a topology on $\mathscr{C}(X)$ for which the standard geometric operations on $\mathscr{C}(X)$ are all continuous (neither $(A, B) \rightarrow \operatorname{cl}(A+B)$ nor 
$(A, B) \rightarrow \operatorname{cl} \operatorname{conv}(A \cup B)$ are continuous on $\mathscr{C}(X) \times \mathscr{C}(X)$, if we equip $\mathscr{C}(X)$ with the Mosco topology). This topology, called the linear topology $\tau_{L}$ in [4], is generated by all sets of the form $V^{-}$where $V$ is norm open, and $H^{\#}$ where $H$ is a closed half space. It is easy to see that "closed half space" could equally well be replaced by "closed hyperplane" or even "closed convex set" in the definition, since two closed convex sets that are a positive distance apart can be strongly separated by a hyperplane. The linear topology has these fundamental analytical characterizations: $(1) \tau_{L}$ is the weakest topology on $\mathscr{E}(X)$ for which both support and distance functionals are continuous with respect to the set argument; (2) $\tau_{L}$ is the weakest topology on $\mathscr{C}(X)$ such that the gap functional

$$
(A, B) \rightarrow \inf \{\|a-b\|: a \in A, b \in B\}
$$

is continuous on $\mathscr{C}(X) \times \mathscr{C}(X)$ (a characterization of $\tau_{M}$ along the same lines is given in Theorem 3.3 of [3]).

Before we introduce our function space topology, we find it convenient to fix a norm on $X \times R$, say $\|\mid(x, \alpha)\| \|=\max \{\|x\|,|\alpha|\}$.

Definition. Let $\Gamma(X)$ be the proper, lower semicontinuous, convex functions on a normed linear space $X$. The affine topology $\tau_{A}$ on $\Gamma(X)$ is the topology generated by all sets of the form $\Gamma(X) \cap V^{-}$where $V$ is norm open in $X \times R$ and $\Gamma(X) \cap(\text { hypo } a)^{\#}$ where $a$ is a continuous real affine function on $X$.

For each open set $V$ in $X \times R, \Gamma(X) \cap V^{-}$consists of those proper 1.s.c. convex functions on $X$ whose epigraphs meet $V$, whereas $\Gamma(X) \cap$ (hypo $a)^{\#}$ consists of those proper l.s.c. convex functions $f$ such that $\inf _{x \in X} f(x)-a(x)>$ 0 . If $a(x)=\langle y, x\rangle-\alpha$, where $y \in X^{\prime}$ and $\alpha \in R$, we shall write $\Omega(y ; \alpha)$ for $(\text { hypo } a)^{\#}$. We shall also need to consider analogous sets of functions defined on the dual $X^{\prime}$ of $X$ : for each $x \in X$ and each $\alpha \in R, \Omega^{\prime}(x ; \alpha)$ will denote all $g \in \Gamma^{*}\left(X^{\prime}\right)$ such that $\inf _{y \in X^{\prime}} g(y)-(\langle y, x\rangle-\alpha)>0$.

Formally, the affine topology $\tau_{A}$ on $\Gamma(X)$ is not stronger than the topology that $\Gamma(X)$ inherits as a subspace of $\left\langle\mathscr{C}(X \times R), \tau_{L}\right\rangle$, for sets of the form $\Gamma(X) \cap H^{\#}$ where $H$ is a vertical half space are not listed as members of the defining subbase for the affine topology. In fact, $\tau_{A}$ is weaker than $\tau_{L}$ on $\Gamma(X)$, as shown by the following example.

Example. On the line, let $f_{n}(x)=n|x|$ and let $f$ be the indicator function of the origin. If $H=\{(x, \alpha): x \geq 1\}$, then $f \in H^{\#}$, whereas $f_{n} \notin H^{\#}$ for each positive integer $n$. To see that $f=\tau_{A}-\lim f_{n}$, suppose $f \in(\text { hypo } a)^{\#}$ where $a(x)=\mu x-\alpha$. Then $\alpha>0$, and it is easy to see that for all $n>|\mu|$, we have $f_{n} \in(\text { hypo } a)^{\#}$, too. That epi $f_{n}$ meets each open set which epi $f$ meets is obvious, for epi $f \subset$ epi $f_{n}$ for each $n \in Z^{+}$.

It is also easy to see that sets of the form (hypo $a)^{\#}$ cannot be replaced by sets of the form $\left((\text { hypo } a)^{c}\right)^{+}$in the definition of the affine topology. 
Example. Again on the line, define $f, f_{1}, f_{2}, \ldots$ by

$$
f(x)=\left\{\begin{array}{ll}
1 / x & \text { if } x>0, \\
\infty & \text { otherwise },
\end{array} \quad f_{n}(x)= \begin{cases}1 / x-1 / n & \text { if } x>0, \\
\infty & \text { otherwise }\end{cases}\right.
$$

It is routine to check that $f=\tau_{A}-\lim f_{n}$. However, if $a$ is the zero functional, we have $f \in\left((\text { hypo } a)^{c}\right)^{+}$, whereas for each $n, f_{n} \notin\left((\text { hypo } a)^{c}\right)^{+}$.

Lemma 2.1. Let $X$ be a normed linear space. Then $\varphi:\left\langle\mathscr{C}(X), \tau_{L}\right\rangle \rightarrow\left\langle\Gamma(X), \tau_{A}\right\rangle$ defined by $\varphi(C)=I(\cdot, C)$ is an embedding.

Proof. We first establish continuity. Let $\pi$ denote the projection map of $X \times R$ onto $X$. Suppose $V$ is norm open in $X \times R$ and $\varphi(C) \in V^{-}$, i.e., epi $I(\cdot, C) \cap$ $V \neq \varnothing$. Then epi $I(\cdot, C) \cap(V \cap(X \times(0, \infty))) \neq \varnothing$, and if $A$ is a nonempty closed convex set with $A \cap \pi(V \cap(X \times(0, \infty))) \neq \varnothing$, then epi $I(\cdot, A) \cap(V \cap(X \times$ $(0, \infty))) \neq \varnothing$. This means that $\pi(V \cap(X \times(0, \infty)))^{-}$is a $\tau_{L}$-neighborhood of $C$ mapped by $\varphi$ into $V^{-}$. Next suppose $\varphi(C) \in \Omega(y ; \alpha)$ for some $y \in X^{\prime}$ and $\alpha \in R$. If $y$ is the zero functional, then $\alpha>0$ must hold, whence $\varphi$ maps of all of $\mathscr{C}(X)$ into $\Omega(y ; \alpha)$. Otherwise, $C \in\{x:\langle y, x\rangle \geq \alpha\}^{\#}$ and $\varphi\left(\{x:\langle y, x\rangle \geq \alpha\}^{\#}\right) \subset \Omega(y ; \alpha)$.

To show that $\varphi$ is relatively open is even easier. It suffices to work with subbasic open sets here, too, since $\varphi$ is one-to-one. If $W$ is open in $X$, then $\varphi\left(W^{-}\right)=\varphi(\mathscr{C}(X)) \cap(W \times R)^{-}$. On the other hand, if $H$ is a closed half space, say $H=\{x:\langle y, x\rangle \geq \alpha\}$, then $\varphi\left(H^{\#}\right)=\varphi(\mathscr{C}(X)) \cap \Omega(y ; \alpha)$.

The proof of the continuity of the Young-Fenchel transform with respect to the Mosco topology $\tau_{M}$ on $\Gamma(X)$ in [5] was based on a presentation of $\tau_{M}$ using a somewhat smaller subbase than the one mentioned above. Specifically, a subbase for $\tau_{M}$ consists of all sets of the form $\Gamma(X) \cap(W \times(-\infty, \alpha))^{-}$where $W$ is norm open in $X$ and $\alpha \in R$, and all sets of the form $\Gamma(X) \cap\left(K^{c}\right)^{+}$where $K$ is a weakly compact subset of $X \times R$ [5, Lemma 2.1]. Exactly the same proof establishes

Lemma 2.2. Let $X$ be a normed linear space. The affine topology $\tau_{A}$ on $\Gamma(X)$ is generated by all sets of the form $\Gamma(X) \cap(W \times(-\infty, \alpha))^{-}$where $W$ is norm open in $X$, and $\Gamma(X) \cap(\text { hypo } a)^{\#}$ where $a$ is a continuous affine functional on $X$.

Since the linear topology is weaker than the usual Hausdorff metric topology on closed convex sets [4, Theorem 3.5], the affine topology $\tau_{A}$ on $\Gamma(X)$ is certainly weaker than the Hausdorff metric topology on $\Gamma(X)$, under the usual identification of functions with their epigraphs. With respect to the Mosco topology, we have

Lemma 2.3. Let $X$ be a normed linear space. Then the affine topology $\tau_{A}$ on $\Gamma(X)$ contains the Mosco topology $\tau_{M}$.

Proof. Suppose $K \subset X \times R$ is weakly compact, $f \in \Gamma(X)$, and epi $f \cap K=\varnothing$. By Lemma 2.2 of [5], there exists continuous real affine functions $a_{1}, \ldots, a_{n}$ on 
$X$ such that for each $i \in\{1, \ldots, n\}, f \in\left(\text { hypo } a_{i}\right)^{\#}$ and $\sup a_{i} \in\left(K^{c}\right)^{+}$. This means that $\bigcap_{i=1}^{n}\left(\text { hypo } a_{i}\right)^{\#}$ is a $\tau_{A}$-neighborhood of $f$ contained in $\left(K^{c}\right)^{+}$. We conclude that $\tau_{A} \supset \tau_{M}$.

The comparative strengths of the topologies $\tau_{M}$ and $\tau_{A}$ are perhaps best indicated by the following simple example: in a normed linear space $X$, if $f_{n}(x)=(1+1 / n)\|x\|$, then $\left\langle f_{n}\right\rangle$ is $\tau_{M}$ and pointwise convergent, but not $\tau_{A^{-}}$ convergent, to $f(x)=\|x\|$ (note: Moscow convergence follows from the weak lower semicontinuity of the norm [6, p. 104]).

In closing this section we show that $\tau_{A}$ is Hausdorff. If $f \in \Gamma(X)$ and $h \in \Gamma(X)$, and $f \neq h$, then we may assume without loss of generality that for some $x_{0} \in X$ we have $f\left(x_{0}\right)<h\left(x_{0}\right)$. Since $h$ is the supremum of the continuous affine functions which it majorizes [6, p. 114], there exists a continuous affine function $a$ on $X$ with $h \in(\text { hypo } a)^{\#}$ and $a\left(x_{0}\right)>f\left(x_{0}\right)$. As a result, (hypo $a)^{\#}$ and $\{(x, \alpha): \alpha<a(x)\}^{-}$are disjoint $\tau_{A^{-}}$-neighborhoods of $h$ and $f$, respectively.

\section{The value functional and the affine topology}

Let $X$ be a normed linear space. We intend to show that $\tau_{A}$ is the weakest topology on $\Gamma(X)$ for which the value functional $f \rightarrow \inf f$ is a continuous functional on $\Gamma(X)$, and such that for each continuous convex function $g$ on $X, f \rightarrow f+g$ is a continuous operator on $\Gamma(X)$. We first show that $\left\langle\Gamma(X), \tau_{A}\right\rangle$ has these properties. The first is trivial; the second requires a "convex algebra" result, perhaps due to Moreau [11], which can be derived as a corollary of the Fenchel-Rockafellar duality theorem [7, §21]. Recall that the epi-sum or infimal convolution of two convex functions on $X$ is defined by the formula

$$
(f \nabla g)(x)=\inf \{f(z)+g(x-z): z \in X\}
$$

Theorem. Let $X$ be a normed linear space, and let $f$ and $g$ be proper l.s.c. convex functions on $X$. Suppose that $g$ is continuous at some point of $\operatorname{dom}(f) \cap$ $\operatorname{int}(\operatorname{dom} g)$. Then $(f+g)^{*}=f^{*} \nabla g^{*}$.

We remark that much stronger results are available when $X$ is a complete normed linear space [2].

Lemma 3.1. Let $X$ be a normed linear space. Then with respect to $\tau_{A}, f \rightarrow$ inf $f$ is a continuous functional on $\Gamma(X)$.

Proof. Suppose $\alpha>\inf f$. This means that for some $x \in X$ we have $f(x)<$ $\alpha$. Then $f \in(X \times(-\infty, \alpha))^{-}$(because $\left.(x, f(x)) \in X \times(-\infty, \alpha)\right)$, and if $h \in(X \times(-\infty, \alpha))^{-}$, then $\inf h<\alpha$. This proves upper semicontinuity of the value functional. Lower semicontinuity of the value functional at $f$ clearly holds if $\inf f=-\infty$. On the other hand, if $-\infty<\beta<\inf f$ and $a(x) \equiv \beta$, then $f \in(\text { hypo } a)^{\#}$, and whenever $h \in(\text { hypo } a)^{\#}$, then $\inf h>\beta$. This proves lower semicontinuity of the value functional. 
Lemma 3.2. Let $X$ be a normed linear space, and let $g$ be a continuous convex real function on $X$. Then with respect to $\tau_{A}, f \rightarrow f+g$ is a continuous operator on $\Gamma(X)$.

Proof. We show that the inverse image of each subbasic open set is open. First, suppose $f+g \in \Omega(y ; \mu)$ where $y \in X^{\prime}$ and $\mu \in R$. Since $\mu>(f+g)^{*}(y)$, by the above result on the conjugate of a sum, there exists $y_{0} \in X^{\prime}$ and $\varepsilon>0$ with $\mu>f^{*}\left(y_{0}\right)+g^{*}\left(y-y_{0}\right)+2 \varepsilon$. Set $\alpha=f^{*}\left(y_{0}\right)+\varepsilon$; then $\Omega\left(y_{0} ; \alpha\right)$ is a $\tau_{A}$-neighborhood of $f$, and whenever $h \in \Omega\left(y_{0} ; \alpha\right)$ and $x \in X$, we have

$$
\begin{aligned}
& (h+g)(x)>\left\langle y_{0}, x\right\rangle-\alpha+\left\langle y-y_{0}, x\right\rangle-g^{*}\left(y-y_{0}\right) \\
& \quad=\langle y, x\rangle-f^{*}\left(y_{0}\right)-\varepsilon-g^{*}\left(y-y_{0}\right) \\
& \quad>\langle y, x\rangle-\mu+\varepsilon .
\end{aligned}
$$

This proves that $h+g \in \Omega(y ; \mu)$, provided $h \in \Omega\left(y_{0} ; \alpha\right)$.

To finish the proof, suppose $f+g \in(W \times(-\infty, \alpha))^{-}$where $W$ is a norm open subset of $X$. Then for some $x \in W$ we have $f(x)+g(x)<\alpha$. Set $\varepsilon=\alpha-$ $f(x)-g(x)$. There exists an open subset $W_{1}$ of $W$ containing $x$ such that for each $w \in W_{1}$, we have $|g(w)-g(x)|<\varepsilon / 2$. Clearly, $\left(W_{1} \times(-\infty, f(x)+\varepsilon / 2)\right)^{-}$ is a $\tau_{A}$-neighborhood of $f$, and if $h \in\left(W_{1} \times(-\infty, f(x)+\varepsilon / 2)\right)^{-}$, there exists $w_{1} \in W_{1}$ with $h\left(w_{1}\right)<f(x)+\varepsilon / 2$. But then $(h+g)\left(w_{1}\right)<(f+g)(x)+\varepsilon$, so that

$$
\left(w_{1},(h+g)\left(w_{1}\right)\right) \in \operatorname{epi}(h+g) \cap\left(W_{1} \times(-\infty, \alpha)\right) .
$$

Thus, $h+g \in(W \times(-\infty, \alpha))^{-}$whenever $h \in\left(W_{1} \times(-\infty, f(x)+\varepsilon / 2)\right)^{-}$.

Example. If $g$ is a fixed proper, lower semicontinuous, convex function, then $f \rightarrow f+g$ need not be $\tau_{A}$-continuous on the continuous real valued convex functions on $X$. To see this, on the line let $g$ be the indicator function of the origin, and for each $n \in Z^{+}$, let $f_{n}(x)=\max \left\{-n^{2} x+n, 0\right\}$.

Theorem 3.3. Let $X$ be a normed linear space and let $\Gamma(X)$ be the proper, lower semicontinuous, convex functions on $X$. Then the affine topology $\tau_{A}$ is the weakest topology $\tau$ on $\Gamma(X)$ such that $f \rightarrow \inf f$ is $\tau$-continuous on $\Gamma(X)$, and for each continuous convex function $g$ on $X, f \rightarrow f+g$ is a $\tau$-continuous operator on $\Gamma(X)$.

Proof. By the last two lemmas, we need only show that if $\tau$ is a topology on $\Gamma(X)$ with these two properties, then $\tau \supset \tau_{A}$. Suppose first that $\tau$ fails to contain $\Gamma(X) \cap(\text { hypo } a)^{\#}$ for some continuous real affine function $a$. There exists $h \in \Gamma(X) \cap(\text { hypo } a)^{\#}$ and a net $\left\langle h_{\lambda}\right\rangle$ in $\Gamma(X) \tau$-convergent to $h$ such that for each $\lambda, h_{\lambda} \notin(\text { hypo } a)^{\#}$. This means that $\inf _{x \in X} h(x)-a(x)>0$, whereas for each index $\lambda, \inf _{x \in X} h_{\lambda}(x)-a(x) \leq 0$. Since $a$ is continuous, we must have $h-a=\tau-\lim h_{\lambda}-a$, and continuity of $f \rightarrow \inf f$ fails at $f=h-a$. This is a contradiction, so that $\tau$ contains all sets of the form (hypo $a$ ) ${ }^{\#}$ whenever $a$ is continuous and affine. 
Now suppose that $\tau$ fails to contain $\Gamma(X) \cap(W \times(-\infty, \alpha))^{-}$for some open $W$ in $X$ and some $\alpha \in R$. There exists $h \in \Gamma(X) \cap(W \times(-\infty, \alpha))^{-}$and a net $\left\langle h_{\lambda}\right\rangle$ in $\Gamma(X) \tau$-convergent to $h$ such that for each $\lambda, h_{\lambda} \notin(W \times(-\infty, \alpha))^{-}$. Choose $x_{0} \in W$ such that $h\left(x_{0}\right)<\alpha$, and a continuous real affine function $a$ with $h \in(\text { hypo } a)^{\#}$ and such that $a\left(x_{0}\right)>h\left(x_{0}\right)-1$. Then $\inf _{x \in X} h(x)-a(x)<$ 1. By the first half of the proof, we may assume without loss of generality that for each index $\lambda, h_{\lambda} \in(\text { hypo } a)^{\#}$. Since $W$ is open, $\alpha>h\left(x_{0}\right)$, and $a$ is continuous, there exist $\varepsilon>0$ and $\delta>0$ such that if $\left\|x-x_{0}\right\|<\varepsilon$, then both $x \in W$ and $a\left(x_{0}\right)+\alpha-h\left(x_{0}\right)-\delta>a(x)$. Let $g$ be the following continuous convex function on $X$ :

$$
g(x)=(1 / \varepsilon) \cdot\left\|x-x_{0}\right\|
$$

We have

$$
\inf _{x \in X}(h-a+g)(x) \leq(h-a+g)\left(x_{0}\right)=(h-a)\left(x_{0}\right)<1 .
$$

We claim that for each index $\lambda$ in our underlying directed set, we have

$$
\inf \left(h_{\lambda}-a+g\right) \geq \min \{1, \inf (h-a+g)+\delta\}>\inf (h-a+g) \text {. }
$$

First, if $\left\|x-x_{0}\right\| \leq \varepsilon$, then both $a\left(x_{0}\right)+\alpha-h\left(x_{0}\right)-\delta>a(x)$ and $x \in W$ hold. Since $g(x) \geq 0$, we obtain

$$
h_{\lambda}(x)+g(x) \geq \alpha+g(x)>a(x)+h\left(x_{0}\right)-a\left(x_{0}\right)+\delta .
$$

Thus, for such $x$,

$$
\left(h_{\lambda}-a+g\right)(x) \geq h\left(x_{0}\right)-a\left(x_{0}\right)+\delta \geq \inf (h-a+g)+\delta .
$$

On the other hand, if $\left\|x-x_{0}\right\|>\varepsilon$ holds, then $g(x) \geq 1$, and since $h_{\lambda} \in$ $(\text { hypo } a)^{\#}$, we obtain

$$
h_{\lambda}(x)-a(x)+g(x)>0+1=1 .
$$

We conclude that $f \rightarrow \inf f$ is not continuous at $f=h-a+g$, completing the proof.

We remark that the proof of Theorem 3.3 can be easily modified to work in the more general setting of a locally convex space, by choosing the function $g$ to be the supremum of an appropriately chosen finite collection of continuous seminorms. We leave the details to the reader.

\section{THE AFFINE TOPOLOGY AND THE YOUNG-FENCHEL TRANSFORM}

In this section we exhibit a topology $\sigma_{A}$ on $\Gamma^{*}\left(X^{\prime}\right)$ "conjugate" to the affine topology $\tau_{A}$ on $\Gamma(X)$, i.e., a topology for which $f \rightarrow f^{*}$ is a homeomorphism of $\left\langle\Gamma(X), \tau_{A}\right\rangle$ onto $\left\langle\Gamma^{*}\left(X^{\prime}\right), \sigma_{A}\right\rangle$. We shall see that when $X$ is reflexive, then this topology is compatible with Mosco plus pointwise convergence of sequences of functions (we do not know whether this is true more generally). We begin with this simple observation, which follows immediately from the definition of the Young-Fenchel transform. 
Lemma 4.1. Let $X$ be a normed linear space. Suppose $f \in \Gamma(X)$. Then for each $y \in X^{\prime}$, we have $f \in \Omega(y ; \alpha)$ if and only if $f^{*} \in(\{y\} \times(-\infty, \alpha))^{-}$, and for each $x \in X$ and $\alpha>0, f \in(\{x\} \times(-\infty, \alpha))^{-}$if and only if $f^{*} \in \Omega^{\prime}(x ; \alpha)$.

The next theorem is in the spirit of the results of Joly [8].

Theorem 4.2. Let $X$ be a normed linear space, and let $\sigma_{A}$ be the topology on $\Gamma^{*}\left(X^{\prime}\right)$ generated by all sets of the form

$$
(\{y\} \times(-\infty, \alpha))^{-}, \quad y \in X^{\prime}, \alpha \in R,
$$

and

$$
\bigcup_{x \in W} \Omega^{\prime}(x ; \alpha), \quad W \text { open in } X, \alpha \in R .
$$

Then $f \rightarrow f^{*}$ is a homeomorphism of $\left\langle\Gamma(X), \tau_{A}\right\rangle$ onto $\left\langle\Gamma^{*}\left(X^{\prime}\right), \sigma_{A}\right\rangle$.

Proof. That the conjugate map is a bijection is well-known [7, p. 46]. Applying Lemma 4.1, for each $y \in X^{\prime}$ and $\alpha>0$, we have

$$
\Omega(y ; \alpha)^{*}=(\{y\} \times(-\infty, \alpha))^{-}
$$

and for each open subset $W$ of $X$ and each $\alpha \in R$, we have

$$
\left((W \times(-\infty, \alpha))^{-}\right)^{*}=\bigcup_{x \in W}\left((\{x\} \times(-\infty, \alpha))^{-}\right)^{*}=\bigcup_{x \in W} \Omega^{\prime}(x ; \alpha) .
$$

In the sequel, we will call the topology $\sigma_{A}$ on $\Gamma^{*}\left(X^{\prime}\right)$ the conjugate affine topology. In a reflexive space, it has a simple presentation.

Theorem 4.3. Let $X$ be a reflexive Banach space. Then the conjugate affine topology on $\Gamma(X)$ is the topology generated by all sets of the form $\left(K^{c}\right)^{+}$where $K$ is a weakly compact subset of $X \times R$, and $(\{x\} \times(-\infty, \alpha))^{-}$where $x \in X$ and $\alpha \in R$.

Proof. By Lemma 2.3, the affine topology on $\Gamma\left(X^{\prime}\right)$ is the weakest topology containing the Mosco topology and all sets of the form $\Omega(x ; \alpha)$ where $x \in X$ and $\alpha \in R$. By Theorem 3.1 of [5], the conjugate operator from $\left\langle\Gamma\left(X^{\prime}\right), \tau_{M}\right\rangle$ to $\left\langle\Gamma(X), \tau_{M}\right\rangle$ is a homeomoprhism, and each set of the form $\Omega(x ; \alpha)$ is mapped to $(\{x\} \times(-\infty, \alpha))^{-}$. As a result, the conjugate affine topology $\sigma_{A}$ on $\Gamma(X)=$ $\Gamma^{*}\left(X^{\prime \prime}\right)$ is generated by all sets of the form

$\left(K^{c}\right)^{+}$where $K$ is a weakly compact subset of $X \times R$,

$(W \times(-\infty, \alpha))^{-}$where $W$ is norm open in $X$ and $\alpha \in R$,

$(\{x\} \times(-\infty, \alpha))^{-}$where $x \in X$ and $\alpha \in R$.

Evidently, the topology generated by all sets of the form $(W \times(-\infty, \alpha))^{-}$where $W$ is norm open in $X$ and $\alpha \in R$ is contained in the one generated by all sets of the form $(\{x\} \times(-\infty, \alpha))^{-}$where $x \in X$ and $\alpha \in R$.

By virtue of the next theorem, in the reflexive setting, $\sigma_{A}$-convergence of a sequence in $\Gamma(X)$ is equivalent to pointwise plus Mosco convergence of the sequence. As Wets and Salinetti have shown [15], pointwise and Mosco convergence of sequences are linked by an equi-lower semicontinuity condition. Such a condition may be expressed in affine terms (see condition (3) below). 
Theorem 4.4. Let $X$ be a reflexive Banach space. Suppose $f \in \Gamma(X)$ and $\left\langle f_{\lambda}\right\rangle$ is a net in $\Gamma(X)$. The following are equivalent:

(1) $f=\tau_{A}-\lim f_{\lambda}$;

(2) $\left\langle f_{\lambda}^{*}\right\rangle$ is pointwise and $\tau_{M}$-convergent to $f^{*}$;

(3) $\left\langle f_{\lambda}^{*}\right\rangle$ is pointwise convergent to $f^{*}$, and whenever $f^{*} \in \Omega(x ; \alpha)$ and $\rho>0$, then for all $\lambda$ sufficiently large and all $y \in X^{\prime}$ with $\|y\| \leq \rho$, we have $f_{\lambda}^{*}(y)>\langle y, x\rangle-\alpha$.

Proof. (1) $\Rightarrow(3)$. We assume $f=\tau_{A}$ - $\lim f_{\lambda}$; by Theorem 4.2, $f^{*}=\sigma_{A}$ - $\lim f_{\lambda}^{*}$. We now use the presentation of $\sigma_{A}$ given in Theorem 4.3. To show pointwise convergence of $\left\langle f_{\lambda}^{*}\right\rangle$ to $f^{*}$, it suffices to show that for each $y \in X^{*}$ and each $\alpha \in R$,

(i) whenever $f^{*}(y)<\alpha$, then $f_{\lambda}^{*}(y)<\alpha$ eventually;

(ii) whenever $f^{*}(y)>\alpha$, then $f_{\lambda}^{*}(y)>\alpha$ eventually.

To prove (i), suppose $f^{*}(y)<\alpha$ holds, i.e., $f^{*} \in(\{y\} \times(-\infty, \alpha))^{-}$. By virtue of $(1)$ and Theorem $4.2, f_{\lambda}^{*} \in(\{y\} \times(-\infty, \alpha))^{-}$eventually, whence $f_{\lambda}^{*}(y)<\alpha$ eventually. To prove (ii), assume $f^{*}(y)>\alpha$. This means that epi $f^{*}$ misses the weakly compact set $\{(y, \alpha)\}$. Again by (1) and Theorems 4.2 and 4.3, the same must be true for epi $f_{\lambda}^{*}$ eventually, so that $f_{\lambda}^{*}(y)>\alpha$ eventually. Pointwise convergence of $\left\langle f_{\lambda}^{*}\right\rangle$ to $f^{*}$ is now established.

Next, suppose that $f^{*} \in \Omega(x ; \alpha)$ and $\rho>0$. Since epi $f^{*}$ lies above the graph of $y \rightarrow\langle y, x\rangle-\alpha$, epi $f^{*}$ fails to meet the intersection $K$ of the graph of $y \rightarrow\langle y, x\rangle-\alpha$ with the cylinder $\{(y, \alpha):\|y\| \leq \rho, \alpha \in R\}$. By reflexivity, $K$ is a weakly compact subset of $X^{\prime} \times R$; so, by Theorems 4.2 and 4.3, epi $f_{\lambda}^{*}$ must miss $K$ eventually. As a result, for all $\lambda$ sufficiently large and for all $y$ with $\|y\| \leq \rho$, we have $\left.f_{\lambda}(y)\right\rangle\langle y, x\rangle-\alpha$.

(3) $\Rightarrow(2)$. Assume condition (3) holds. By pointwise convergence, if epi $f^{*}$ meets $W \times(-\infty, \alpha)$ where $W$ is open in $X^{\prime}$, then the same is true for epi $f_{\lambda}^{*}$ eventually. Now suppose epi $f^{*}$ misses some weakly compact subset $K$ of $X^{\prime} \times R$. Choose $\rho>0$ so large that the projection of $K$ onto $X^{\prime}$ is contained in the ball of radius $\rho$ about the origin. Let $a_{1}, a_{2}, \ldots, a_{n}$ be continuous real affine functions on $X^{\prime}$ such that $\operatorname{epi}\left(\sup a_{i}\right) \cap K=\varnothing$ and for all $i \leq n$, $\inf _{y \in X^{\prime}} f(y)-a_{i}(y)>0$. By condition (3), there exists $\lambda_{0}$ in the underlying directed set for the net such that for all $\lambda \geq \lambda_{0}$ and all $i \leq n$, we have $f_{\lambda}(y)>$ $a_{i}(y)$, provided $\|y\| \leq \rho$. Since $\operatorname{epi}\left(\sup a_{i}\right) \cap K=\varnothing$, for all $\lambda \geq \lambda_{0}$, we have

$$
\text { epi } f_{\lambda} \cap K \cap\{(y, \alpha):\|y\| \leq \rho \text { and } \alpha \in R\}=\varnothing .
$$

The choice of $\rho$ ensures that $f_{\lambda} \in\left(K^{c}\right)^{+}$whenever $\lambda \geq \lambda_{0}$. As a result, $f=\tau_{M}-\lim f_{\lambda}$.

(2) $\Rightarrow(1)$. Pointwise plus Mosco convergence of $\left\langle f_{\lambda}^{*}\right\rangle$ to $f^{*}$ evidently force convergence in the topology $\sigma_{A}$ on $\Gamma\left(X^{\prime}\right)$ as described by Theorem 4.3. Condition (1) now follows from Theorem 4.2. 
From Theorem 4.4, it is evident that the affine and conjugate affine topologies are noncomparable, even when $X$ is the line. We have already noted in $\S 2$ that pointwise plus Mosco convergence of continuous convex functions on the line does not ensure $\tau_{A}$-convergence. On the other hand, if $g_{n}(x)=$ $\max \left\{-n^{2} x+n, 0\right\}$, then $\left\langle g_{n}\right\rangle$ is $\tau_{A}$-convergent, but not $\sigma_{A}$-convergent, because $\lim _{n \rightarrow \infty} g_{n}(0) \neq 0$.

\section{REFERENCES}

1. H. Attouch, Variational convergence for functions and operators, Pitman, Boston, Mass., 1984.

2. H. Attouch and H. Brezis, Duality for the sum of convex functions in general Banach spaces, AVAMAC, Université de Perpignan, 1984.

3. G. Beer, On Mosco convergence of convex sets, Bull. Austral. Math. Soc. 38 (1988), 239-253.

4. $\ldots$, Support and distance functionals for convex sets, Numer. Funct. Anal. Optim. 10 (1989), 15-36.

5. $\ldots$, On the Young-Fenchel transform for convex functions, Proc. Amer. Math. Soc. 104 (1988), 1115-1123.

6. J. Giles, Convex analysis with application in differentiation of convex functions, Research Notes in Math., vol. 58, Pitman, London, 1982.

7. R. Holmes, A course in optimizaiton and best approximation, Lecture Notes in Math., vol. 257, Springer-Verlag, New York, 1972.

8. J. Joly, Une famille de topologies sur l'ensemble des fonctions convexes pour lesquelles la polarité est bicontinue, J. Math. Pures Appl. 52 (1973), 421-441.

9. E. Klein and A. Thompson, Theory of correspondences, Wiley, Toronto, 1984.

10. E. Michael, Topologies on spaces of subsets, Trans. Amer. Math. Soc. 71 (1951), 152-182.

11. J.-J. Moreau, Inf-Convolution des fonctions numériques sur un espace vectoriel, C. R. Acad. Sci. Paris 256 (1963), 5047-5049.

12. U. Mosco, Convergence of convex sets and of solutions of variational inequalitites, Adv. in Math. 3 (1969), 510-585.

13. $\frac{1}{535}$, On the continuity of the Young-Fenchel transform, J. Math. Anal. Appl. 35 (1971), 518535.

14. R. Robert, Convergence de fonctionelles convexes, J. Math. Anal. Appl. 45 (1974), 533-555.

15. G. Salinetti and R. Wets, On the relationship between two types of convergence for convex functions, J. Math. Anal. Appl. 60 (1977), 211-226.

16. D. Walkup and R. Wets, Continuity of some convex-cone valued mappings, Proc. Amer. Math. Soc. 18 (1967), 229-235.

17. R. Wijsman, Convergence of sequences of convex sets, cones, and functions. II, Trans. Amer. Math. Soc. 123 (1966), 32-45.

Department of Mathematics, California State University, Los Angeles, California 